Pacific

Journal of

Mathematics

EXISTENCE OF WEAK SOLUTIONS TO A CLASS OF NONSTRICTLY HYPERBOLIC CONSERVATION LAWS WITH NON-INTERACTING WAVES

Anthony J. Kearsley And Andrea M. Reiff

Volume $205 \quad$ No. 1

July 2002 


\title{
EXISTENCE OF WEAK SOLUTIONS TO A CLASS OF NONSTRICTLY HYPERBOLIC CONSERVATION LAWS WITH NON-INTERACTING WAVES
}

\author{
Anthony J. Kearsley and Andrea M. Reiff
}

\begin{abstract}
Many applied problems resulting in hyperbolic conservation laws are nonstrictly hyperbolic. As of yet, there is no comprehensive theory to describe the solutions of these systems. In this paper, a proof of existence is given for a class of nonstrictly hyperbolic conservation laws using a proof technique first applied by Glimm to systems of strictly hyperbolic conservation laws. We show that Glimm's scheme can be used to construct a subsequence converging to a weak solution. This paper necessarily departs from previous work in showing the existence of a convergent subsequence. A novel functional, shown to be equivalent to the total variation norm, is defined according to wave interactions. These interactions can be bounded without any assumptions of strict hyperbolicity.
\end{abstract}

\section{Introduction.}

A conservation law is called nonstrictly hyperbolic if the eigenvalues of the Jacobian of the flux function are not distinct. There are many conservation laws which are nonstrictly hyperbolic: Including the multicomponent gas dynamics equations, the polytropic three-dimensional Euler gas dynamics equations, the equations modeling an elastic string in the plane, models which appear in magnetohydrodynamics, and cavitating liquid-vapor mixtures, to name a few. Unlike the strictly hyperbolic problem, there is no general theory for existence of weak solutions to nonstrictly hyperbolic problems. In addition, there are no general results describing the asymptotic decay of the associated Cauchy problem.

Nonstrictly hyperbolic problems can be classified into two types. The first class is characterized by a flux function whose Jacobian possesses eigenvalues of constant multiplicity greater than one. The second class consists of problems possessing eigenvalues which coalesce on a subset of phase space. In this case, the problem may exhibit a parabolic degeneracy. We say that a conservation law exhibits a parabolic degeneracy if, at some point of phase space, the Jacobian of the flux function possesses an eigenvector deficiency. 
Keyfitz and Kranzer studied a number of model problems of this second type (see [8] and [9]).

Here, we examine a conservation law belonging to the first class of nonstrictly hyperbolic conservation laws; that is, those exhibiting non-simple eigenvalues of constant multiplicity. In particular, we consider the problem

$$
U_{t}+(\Theta U)_{x}=0,
$$

where $\Theta=\Theta(U)$ is a strictly convex and continuously differentiable function of $U$. We show that for the initial data

$$
0<c_{1} \leq\left\|U_{0}\right\| \leq c_{2},
$$

there exists a weak solution of the conservation law when the initial data is contained in an open half-space. The proof technique is similar to the classical one introduced by Glimm. Unlike the classical proof by Glimm, there is no assumption that the total variation of the initial data be small.

\section{Preliminaries.}

Let $x \in \Re, u(x, t) \in \Re^{n}$, and $f(u) \in \Re^{n}$, we say that the conservation law given by:

$$
u_{t}+f(u)_{x}=0,
$$

is called hyperbolic if the Jacobian of $f(u)$ is diagonalizable. If the $n$ eigenvalues of the Jacobian of $f$ are real and distinct, we say that (3) is strictly hyperbolic. If the eigenvalues are real but not everywhere distinct, we say that (3) is nonstrictly hyperbolic.

Consider the conservation law system (3) where $\lambda_{i}$ is an eigenvalue of the Jacobian of $f$ with $r_{i}$ an associated eigenvector. The Riemann problem for the conservation law (3) is defined to be the differential equation (3) along with initial data:

$$
u(x, 0)=u_{0}= \begin{cases}u_{L} & \text { for } x<0 \\ u_{R} & \text { for } x>0 .\end{cases}
$$

Definition 2.1. The $i$-th characteristic family is said to be genuinely nonlinear if $\nabla \lambda_{i} \cdot r_{i} \neq 0$.

There are two (classical) types of nonlinear waves: Centered rarefaction waves and shock waves. Rarefaction waves are continuous solutions to the differential equation, while shock waves are discontinuous solutions. We first discuss the rarefaction waves.

Since a centered rarefaction wave is a continuous self-similar solution, we perform the following change of variables $\xi=x / t$. With the change of variables, Equation (3) becomes

$$
(d f(u)-\xi I) u_{\xi}=0,
$$


which implies $\xi$ is an eigenvalue of $d f$ and $u_{\xi}$ is the corresponding eigenvector. Thus, $\xi=\lambda_{i}(u(\xi))$ and $u_{\xi}=r_{i}(u(\xi))$ for some $i$. We say that $u_{L}$ is connected to $u_{R}$ by a $k$-rarefaction wave if

$$
\begin{gathered}
\lambda_{k}\left(u_{R}\right)>\lambda_{k}\left(u_{L}\right), \\
\text { and } \lambda_{k}(u(\xi))=\xi \text { for } \lambda_{k}\left(u_{L}\right) \leq \xi \leq \lambda_{k}\left(u_{R}\right) .
\end{gathered}
$$

Now consider a shock wave solving the Riemann problem. A $k$-shock is a discontinuous solution which satisfies the Rankine-Hugoniot relation:

$$
s\left(u_{R}-u_{L}\right)=\left(f\left(u_{R}\right)-f\left(u_{L}\right)\right),
$$

where $s$ is the speed at which the shock travels. In addition, the $k$-shock must satisfy the Lax entropy condition,

$$
\lambda_{k-1}\left(u_{L}\right)<s<\lambda_{k}\left(u_{L}\right) \text { and } \lambda_{k}\left(u_{R}\right)<s<\lambda_{k+1}\left(u_{R}\right) .
$$

In this case, the solution to the Riemann problem is

$$
u(x, t)= \begin{cases}u_{L} & \text { for } x<s t \\ u_{R} & \text { for } x>s t .\end{cases}
$$

Having defined the possible nonlinear waves, we now define a linear wave.

Definition 2.2. The $i$-th characteristic family is said to be linearly degenerate if $\nabla \lambda_{i} \cdot r_{i} \equiv 0$.

Considering the Riemann problem, we say that the two states $u_{L}$ and $u_{R}$ are connected by a $k$-contact discontinuity if $\lambda_{k}\left(u_{L}\right)=\lambda_{k}\left(u_{R}\right)$. Here, the solution is given by

$$
u(x, t)= \begin{cases}u_{L} & \text { for } x<s t \\ u_{R} & \text { for } x>s t\end{cases}
$$

where the propagation speed is $s=\lambda_{k}\left(u_{L}\right)$.

For a fixed state $u_{L}$, let $S_{k}$ be the curve in state space of all the states which can be connected to $u_{L}$ on the left by a $k$-shock, let $R_{k}$ be the curve containing all the states which can be connected to $u_{L}$ on the left by a $k$ rarefaction wave, and let $C_{k}$ be the subset containing all the states which can be connected to $u_{L}$ by a $k$-contact discontinuity. The locus at state $u_{L}$ is defined to be the union of all these curves $R_{i}, S_{i}$, and $C_{i}$ for all $i$. Using the wave loci, we are able to determine the solution to the Riemann problem in a neighborhood of $u_{L}$.

We shall now fix notation which will be used throughout the paper.

(i) For $V \in \Re^{m}$, let $\|V\|$ denote the Euclidean norm of $V$.

(ii) For $V(x): \Re \rightarrow \Re^{m}$, let $\|V\|_{T V}$ be the total variation of $V$.

(iii) Let $\left.U\right|_{S}$ denote the restriction of $U$ to the set $S$. 


\section{The Riemann problem.}

In this section, we show that the Riemann problem for (1) is always solvable when the initial states are in an open half-space and are bounded away from the umbilic point at the origin. Before discussing the solution to the general Riemann problem, we discuss the structure of the Rankine-Hugoniot locus.

First, we analyze those states which can be connected by a nonlinear wave. We begin with those states $U$ which can be connected to $U_{L}$ by a shock wave. From the Rankine-Hugoniot relation, we obtain

$$
U=\left(\frac{s-\Theta_{L}}{s-\Theta(U)}\right) U_{L}
$$

where $s$ is the speed of the shock wave and $\Theta_{L}=\Theta\left(U_{L}\right)$. Clearly, the states connected by a shock wave are necessarily on the same radial line. The Lax entropy conditions give the result

$$
\frac{s-\Theta_{L}}{s-\Theta(U)}>0
$$

Furthermore, the strict convexity of $\Theta(U)$, and the fact that the states lie on the same radial line, imply that $\|U\|<\left\|U_{L}\right\|$.

The other possible nonlinear wave connecting $U$ to $U_{L}$ is a rarefaction wave. Since rarefaction waves are continuous solutions to the conservation law, we can perform the change of variables $\xi=x / t$ and obtain the following differential equation from (1),

$$
(d(\Theta U)-\xi I) U_{\xi}=0 .
$$

Assuming that $U_{\xi} \neq 0$, it must be the case that $U_{\xi}=r_{2}$, where $r_{2}=U$ is an eigenvector associated with $\lambda_{2}$. Integrating $r_{2}$ shows that the states connected by rarefaction waves are also on the same radial line as $U_{L}$. Moreover, as a rarefaction wave, the inequality $\lambda_{2}\left(U_{L}\right)<\lambda_{2}(U)$ must hold. This, along with our assumptions on $\Theta$, implies that $\left\|U_{L}\right\|<\|U\|$; this, since the two states are on the same radial line.

Let us now consider the linear waves and analyze which states can be connected to $U_{L}$ on the left by a contact discontinuity. For any state $U$ connected to $U_{L}$ by a contact discontinuity, with $\lambda_{1}(U)=\lambda_{1}\left(U_{L}\right)$, it follows that $U$ must lie on the same level set of $\lambda_{1}=\Theta$ as $U_{L}$. Since we assume $\Theta(U)$ is sufficiently smooth, the tangent space of $\Theta(U)$ is well-defined.

Unique solutions to the Riemann problem are desirable. However, it is not clear which Riemann solution is correct for data $U_{L}$ and $U_{R}$ in the event that $U_{R}=-\mid$ Const $\mid U_{L}$ and $\lambda_{1}\left(U_{L}\right)=\lambda_{1}\left(U_{R}\right)$. Since the two states belong to the same level set of $\lambda_{1}=\Theta$, one possible solution is that the two states are joined by a contact discontinuity. However, because the two states are on the same radial line, another possible solution is a composite solution of 
a shock and an adjacent rarefaction wave. This can be observed by writing the conservation law (1) as the scalar conservation law

$$
\left(a U_{L}\right)_{t}+\left(\Theta\left(a U_{L}\right) a U_{L}\right)_{x}=0,
$$

where $a=a(x, t)$, with initial data

$$
a(x, 0) U_{L}= \begin{cases}U_{L} & \text { for } x<0 \\ U_{R} & \text { for } x>0 .\end{cases}
$$

The composite solution is discussed in [5] for $m=2$ and $\Theta$ a symmetric function of $U$. This difficulty is avoided by requiring that the data $U_{L}$ and $U_{R}$ are such that the angle between the two states is strictly smaller than $\pi$. Let $\Omega$ consist of the rays, or half-lines, containing the initial data $U_{L}$ and $U_{R}$ so that

$$
\Omega \equiv\left\{r U_{L}: r>0\right\} \cup\left\{r U_{R}: r>0\right\} .
$$

Note that if $U_{L}$ and $U_{R}$ are contained in an open half-space, then $\Omega$ is also contained in an open half-space.

Having developed an understanding of the Hugoniot locus, we are prepared to solve the general Riemann problem. Here we will need the assumption that

$$
U_{R} \neq-\mid \text { Const } \mid U_{L}
$$

along with the assumption that neither state is the zero state. Given these two assumptions, there are four possibilities for the initial data. We consider these four cases individually.

(i) Case 1: $U_{R}=r U_{L}$. Because the two states are on the same radial line, they must be connected by a nonlinear wave. If $r>1$ or $r \in(0,1)$, then $U_{R}$ is connected to $U_{L}$ on the left by a rarefaction or shock wave, respectively. In the case of a shock wave, the convexity of $\Theta$ implies $\Theta\left(U_{R}\right)<\Theta\left(U_{L}\right)$. The speed of the shock wave, s, is given by

$$
s=\frac{r \Theta\left(r U_{L}\right)-\Theta_{L}}{(r-1)} .
$$

In the case of a rarefaction wave, the solution $U$ is on the same radial line as $U_{L}$ and $U_{R}$, and by the convexity of $\Theta$, obeys $\Theta\left(U_{R}\right) \geq \Theta(U) \geq$ $\Theta\left(U_{L}\right)$. For a rarefaction wave, the fastest part of the wave travels with speed $\lambda_{2}\left(U_{R}\right)$, the slowest with speed $\lambda_{2}\left(U_{L}\right)$.

(ii) Case 2: $\Theta\left(U_{R}\right)=\Theta\left(U_{L}\right)$. The two states are on the same level set of $\Theta$, so $U_{R}$ is connected to $U_{L}$ on the left by a contact discontinuity. Here, the speed of the linear wave is given by $s=\Theta\left(U_{L}\right)$.

(iii) Case 3: $\Theta\left(U_{R}\right)>\Theta\left(U_{L}\right)$ and $U_{R} \neq r U_{L}$. There exists an intermediate state $U_{I}$, where $U_{I}$ is connected to $U_{L}$ on the left by the slower linear wave and to $U_{R}$ on the right by the faster nonlinear wave. Thus, $\Theta\left(U_{I}\right)=\Theta\left(U_{L}\right)<\Theta\left(U_{R}\right)$ and $U_{I}=r U_{R}$ for some $r<1$. Since 
$U_{I}=r U_{R}$ for $r \in(0,1), U_{R}$ is connected to $U_{I}$ by a rarefaction wave. Using the assumption that $\Theta$ is strictly convex with minimum value at the origin, the function $\Theta\left(r U_{R}\right)$ is strictly monotone increasing for $r \in(0,1)$. Thus, a unique value of $U_{I}=r U_{R}$ satisfying $\Theta\left(U_{I}\right)=\Theta\left(U_{L}\right)$ exists. We now have that the solution to the Riemann problem consists of a contact discontinuity connecting state $U_{L}$ to $U_{I}$, followed by a rarefaction wave connecting state $U_{I}$ to $U_{R}$.

(iv) Case 4: $\Theta\left(U_{R}\right)<\Theta\left(U_{L}\right)$ and $U_{R} \neq r U_{L}$. There must be an intermediate state $U_{I}$ connected to $U_{L}$ on the left by the slower linear wave and, then, to $U_{R}$ on the right by the faster nonlinear wave. Thus, $\Theta\left(U_{I}\right)=\Theta\left(U_{L}\right)>\Theta\left(U_{R}\right)$ and $U_{I}=r U_{R}$ for some $r>1$. So $U_{L}$ is connected to $U_{R}$ first by a contact discontinuity to an intermediate state $U_{I}$ and then by a shock wave. As in Case 3, the value of $U_{I}$ is unique.

From the four cases above, we make two important observations: (i) Riemann solutions remain in $\Omega$, the set of half-lines containing initial data $U_{R}$ and $U_{L}$, and (ii) any Riemann solution $U$ is bounded by $\Theta\left(U_{L}\right)$ and $\Theta\left(U_{R}\right)$. We have now completely characterized the solutions to the Riemann problem. The solution to a Riemann problem is used as a fundamental step in many algorithms to approximate the solution to a Cauchy problem. Glimm's scheme, sometimes referred to as the random choice method, is one algorithm which relies on the solutions of Riemann problems. In what follows, we will employ Glimm's scheme (see [6]) to demonstrate the existence of weak solutions to Equation (1) for initial data $U(x, 0) \equiv U_{0}(x)$ which is contained in a half-space, and satisfies (2) and

$$
\left\|U_{0}\right\|_{T V}<M_{0} \text {. }
$$

The method of proof follows the classical technique for proving existence of weak solutions for strictly hyperbolic conservation laws introduced by Glimm in [6]. Glimm's random choice scheme is employed to generate a sequence of approximate solutions, which will then be shown to possess a weak solution as an accumulation point.

\section{Glimm's Scheme.}

Consider the Cauchy problem for a system of conservation laws

$$
v_{t}+f(v)_{x}=0
$$

with

$$
v(x, 0)=v_{0}(x) .
$$

Given some sufficiently small value of time, say $t^{\prime}$, an approximation to $v(x, t)$ at time $t^{\prime}$ is given by the solution to the conservation law system

$$
v_{t}+f(v)_{x}=0,
$$


with

$$
v(x, 0)=\widetilde{v}_{0}(x),
$$

where $\widetilde{v}_{0}$ is a piecewise constant approximation to the initial data $v_{0}$. An approximate solution to the problem is constructed by solving a Riemann problem at every discontinuity in $\widetilde{v}_{0}$. This process forms the fundamental basis for each time step of Glimm's scheme. Let $l=\Delta x$ be a discretization of space and $k=\Delta t$ be a discretization of time. Glimm's scheme generates an approximate solution at $(x, t)$, say $u_{h}(x, t)$, where the mesh size $h=(l, k)$. Suppose that $u_{h}(x,(n-1) k)$ is constant on the interval $[2 m l, 2(m+1) l]$ for every integer $m$. For $t \in[(n-1) k, n k]$, let $v(x, t)$ be the solution to the resulting Riemann problems on intervals $[(2 m-1) l,(2 m+1) l]$, with initial data $v(x,(n-1) k)=u_{h}(x,(n-1) k)$. For $t \in[(n-1) k, n k)$, we choose the approximation $u_{h}(x, t) \equiv v(x, t)$. Hence, between time steps $n-1$ and $n$, the approximate solution, $u_{h}(x, t)$, is the exact solution to Riemann problems solved at the cell interfaces of the previous time step $n-1$. At time $t=n k$, the approximation $u_{h}(x, n k)$ is chosen to be a piecewise constant function approximating the solution $v(x, t)$ at time $t=n k$, with constant values selected using a random choice. Let $\theta \equiv\left\{\theta_{i}\right\}$ be a sequence of random numbers from a uniform distribution on $[-1,1]$. If $v(x, t)$ is the solution to the Riemann problem with initial data $u_{h}(x,(n-1) k)$, then $u_{h}(x, n k)$ is chosen to be constant on the interval $[(2 m-1) l,(2 m+1) l]$, with constant value equal to $v\left(\left(2 m+\theta_{n}\right) l, n k\right)$. The sequence $\left\{\theta_{i}\right\}$ contains the sampling points which are used to construct the piecewise constant approximation $u_{h}(x, n k)$, for all integer $n$.

To avoid wave interactions between Riemann problems, it is necessary to choose the ratio $k / l$ to be smaller that the reciprocal of the largest possible wave speed. This condition is commonly known as the Courant-FredrichsLewy condition or CFL condition (see for instance [10] and [11] among numerous other references). The following is an algorithmic description of Glimm's Scheme. A more detailed description can be found in the paper by Glimm (see [6]).

\section{Glimm's Scheme}

(i) Initialize $u_{h}(x, 0)$ to be piecewise constant. For $x$ in the interval $[(2 m-$ 1) $l,(2 m+1) l]$, set $u_{h}(x, 0)=u_{0}\left(\left(2 m+\theta_{0}\right) l\right)$.

(ii) For $n=1,2, .$. define $u_{h}(x, t)$ in the $n$-th time interval. When $m+n$ is even, solve the following Riemann problem:

$$
\begin{gathered}
v_{t}+f(v)_{x}=0, \\
v(x,(n-1) k)= \begin{cases}u_{h}((m-1) l,(n-1) k) & \text { for } x<m l \\
u_{h}((m+1) l,(n-1) k) & \text { for } x>m l .\end{cases}
\end{gathered}
$$

Set $u_{h}(x, t) \equiv v(x, t)$ when $x \in[(m-1) l,(m+1) l]$ and $t \in((n-1) l, n k)$. 
(iii) Choose piecewise constant data for the next time step. Choose $u_{h}(x, n k)$ to have constant value $u_{h}(x, n k)=v\left(\left(m+\theta_{n}\right) l, n k\right)$ on the interval $[(m-1) l,(m+1) l]$, when $m+n$ is even.

At any time $t=n k$, Glimm's scheme generates an approximate solution $u_{h}(x, n k)$ which is constructed to be constant on every interval (or cell) $[(m-1) l,(m+1) l]$ where $m+n$ is even. Cell interfaces, or jumps in the piecewise constant $u_{h}(x, n k)$, occur only at $x=m l$ where $m+n$ is odd.

Consider the set $\left\{a_{m, n}\right\}$ consisting of the randomly sampled points $a_{m, n} \equiv$ $\left(\left(m+\theta_{n}\right) l, n k\right)$ with $m+n$ even. The set $\left\{a_{m, n}\right\}$ contains the points that are sampled to determine the piecewise constant approximation $u_{h}$ at each time step $n$. Note that we can consider $\left\{a_{m, n}\right\}$ as forming a discrete mesh. Since it will later be of value to estimate wave interactions across this mesh, we now introduce the following definition.

Definition 4.1. A mesh curve is a piecewise linear curve consisting of a set of mesh points and the line segments joining them, where, if $a_{m, n}$ belongs to the curve, then either $a_{m+1, n-1}$ or $a_{m+1, n+1}$ belongs to the curve but not both.

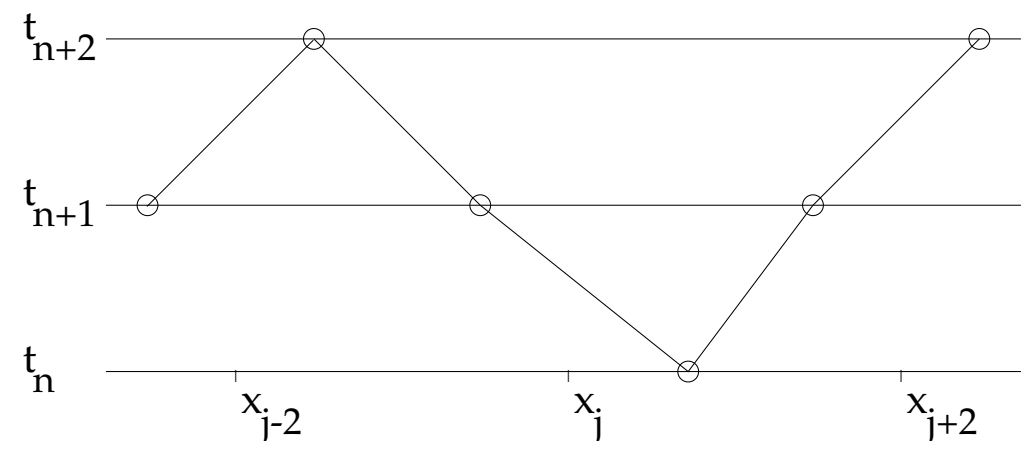

Figure 1. A mesh curve.

We introduce a partial order " $\succ$ " on mesh curves, as follows. We say the mesh curve $I_{1} \succ I_{2}$ if every point of $I_{1}$ is a point of $I_{2}$ or lies above the mesh curve $I_{2}$. A mesh curve $I$ is called an immediate successor to $J$ if $I \succ J$ and every mesh point of $I$ except one is on $J$. In addition, we let $\mathcal{O}$ be the unique mesh curve which passes through the mesh points on $t=0$ and $t=\Delta t$.

\section{Bounds on Glimm's solutions.}

The proof is broken down into two components: First we show that the Glimm scheme produces a convergent subsequence of approximate solutions 
and second, we demonstrate that this subsequence converges to a weak solution. At this point it is not yet clear that the Riemann problems are uniquely solvable at every time step of the Glimm scheme. For the conservation law system (1), Riemann solutions are unique for data in an open half-space, and it is sufficient that the solutions $\left\{U_{h}\right\}$ generated by the Glimm scheme satisfy $U_{h} \in \Omega, \Omega$ a domain in a half-space. In the process of proving that the Glimm scheme produces a convergent subsequence, we give bounds on the approximate solutions showing that the solutions do in fact remain in a half-space.

The proof that Glimm's scheme produces a convergent subsequence relies heavily on the use of Helly's theorem. Loosely stated, Helly's theorem guarantees that if a set of functions is both uniformly bounded and has uniformly bounded total variation, then it is relatively compact and, hence, contains a convergent subsequence (see [2]). Thus, in order to use this result, we show that the functions $\left\{U_{h}\right\}$ generated by Glimm's scheme satisfy the following:

$$
C_{1} \leq\left\|U_{h}\right\| \leq C_{2}
$$

and

$$
\left\|U_{h}(\cdot, \tau)\right\|_{T V}<M_{1},
$$

where $C_{1}, C_{2}$, and $M_{1}$ are independent of the mesh size $h$.

We will proceed to show that the sequence generated by Glimm's scheme is bounded and has bounded variation. We accomplish this by showing that the sequence is uniformly bounded in a functional equivalent to the total variation. This, in turn, is done by demonstrating that wave interactions will not cause an increase in the total variation. We will measure the "strength" of these wave interactions using the solutions of the fundamental Riemann problem.

We first define the wave strengths of the Riemann solutions. Suppose that $U_{k-1}$ and $U_{k}$ are the states to the left and right of a $k$-wave. Define $\epsilon_{k}$, the wave strength of the $k$-wave connecting the states $U_{k-1}$ to $U_{k}$, where the $k$-wave is either a shock, a rarefaction wave, or a contact discontinuity.

For the conservation law system (1), we define the wave strengths as follows:

(i) If the states $U_{l}$ and $U_{r}$ are connected by the faster nonlinear wave (a shock or a rarefaction), we will choose the strength of the wave to be $\epsilon_{2}=\Theta\left(U_{r}\right)-\Theta\left(U_{l}\right)$. (Note that $\epsilon_{2}<0$ in the case of a shock and $\epsilon_{2}>0$ in the case of a rarefaction.)

(ii) If the states $U_{l}$ and $U_{r}$ are connected by the slower linear wave (a contact discontinuity), we will choose the strength of the wave to be $\epsilon_{1}=\arccos \left(\frac{U_{l} \cdot U_{r}}{\left\|U_{l}\right\|\left\|U_{r}\right\|}\right)$. Thus, $\epsilon_{i} \in[0, \pi]$ is the angle between $U_{l}$ and $U_{r}$. 
We now proceed to look at interactions involving three states $U_{l}, U_{r}$, and $U_{m}$. Assume that the three states $U_{l}, U_{r}$, and $U_{m}$ are bounded away from zero and that all three are contained in an open half-space. In this way, we guarantee that the states are sufficiently close that the angle between any two of the states is smaller than $\pi$. Denote by

$$
\left(U_{l}, U_{r}\right)=\left[\left(U_{l}=U_{0}, U_{1}, U_{2}=U_{r}\right) /\left(\epsilon_{1}, \epsilon_{2}\right)\right],
$$

the solution to the Riemann problem with initial data:

$$
U(x, 0)= \begin{cases}U_{l} & \text { if } x<0 \\ U_{r} & \text { if } x>0\end{cases}
$$

For the solutions to the Riemann problems involving $U_{l}, U_{m}$ and $U_{m}, U_{r}$, respectively, we have

$$
\begin{aligned}
& \left(U_{l}, U_{m}\right)=\left[\left(U_{l}=U_{0}^{\prime}, U_{1}^{\prime}, U_{2}^{\prime}=U_{m}\right) /\left(\gamma_{1}, \gamma_{2}\right)\right], \\
& \left(U_{m}, U_{r}\right)=\left[\left(U_{m}=U_{0}^{\prime \prime}, U_{1}^{\prime \prime}, U_{2}^{\prime \prime}=U_{r}\right) /\left(\delta_{1}, \delta_{2}\right)\right] .
\end{aligned}
$$

We say that the $j$-wave $\gamma_{j}$ and the $k$-wave $\delta_{k}$ are approaching waves

(i) if $j>k$, or

(ii) if $j=k$ and at least one of the waves is a shock.

Proposition 5.1. If $U_{l}, U_{m}, U_{r}$ are three states with the wave strengths defined by (7)-(9), then

$$
\epsilon_{1} \leq \gamma_{1}+\delta_{1}
$$

and

$$
\epsilon_{2}=\gamma_{2}+\delta_{2}
$$

The proof follows from the geometric construction of the wave strengths and a compatibility condition with the behavior of the linear and nonlinear waves. We also observe that wave interactions between the two families completely decouple.

Now, we introduce a functional $\mathcal{L}(J)$, which we will show is equivalent to the total variation norm. Letting $J$ be a mesh curve and $\epsilon$ a wave in the approximate solution $U_{h}$, define

$$
\mathcal{L}(J)=\sum\{|\epsilon|: \epsilon \text { crosses } J\} .
$$

The following lemma and proof are a simplification of the one given in the book by Smoller ([13]). Since wave interactions decouple, there is no need for quadratic terms as in Glimm's original proof.

Lemma 5.2. Let $J$ be any mesh curve which is in the domain of definition of $U_{h} ;$ then $\mathcal{L}(J) \leq \mathcal{L}(\mathcal{O})$. 
Proof. Suppose that $I$ is in the domain of definition of $U_{h}$. Assume $J$ is an immediate successor to $I$; hence, $J$ and $I$ differ by a single diamond.

Let $I=I_{o} \cup I^{\prime}$ and $J=I_{o} \cup J_{o}$. Note that

$$
\mathcal{L}(I)=\mathcal{L}\left(I_{o}\right)+\sum_{I^{\prime}}\left|\gamma_{i}\right|+\sum_{I^{\prime}}\left|\delta_{i}\right|,
$$

and

$$
\mathcal{L}(J)=\mathcal{L}\left(I_{o}\right)+\sum_{J_{o}}\left|\epsilon_{i}\right| .
$$

It follows from Proposition 5.1 that

$$
\mathcal{L}(J)=\mathcal{L}\left(I_{o}\right)+\sum_{J_{o}}\left|\epsilon_{i}\right| \leq \mathcal{L}\left(I_{o}\right)+\sum_{I^{\prime}}\left(\left|\gamma_{i}\right|+\left|\delta_{i}\right|\right)=\mathcal{L}(I) .
$$

Hence, $\mathcal{L}$ is a monotone decreasing function relative to the partial order on the mesh curves. For any mesh curve $J$, we have $J \succ \mathcal{O}$, then it follows that $\mathcal{L}(J) \leq \mathcal{L}(\mathcal{O})$. This observation concludes the proof.

We now show that choosing the initial data to have bounded total variation implies that $\mathcal{L}(\mathcal{O})$ is also bounded.

Lemma 5.3. Assume that $U_{0}$ is contained in an open half-space and satisfies (2). There exists a constant $\mathcal{K}$, independent of the size of the mesh, so that

$$
\mathcal{K}\left\|U_{0}\right\|_{T V}>\mathcal{L}(\mathcal{O}),
$$

where $\mathcal{O}$ is the unique mesh curve between $t=0$ and $\Delta t$.

Proof. Let $U_{h}(x, 0)=\bar{U}_{0}$, a piecewise constant approximation to the data $U_{0}$. We observe that the $\epsilon$-waves crossing mesh curve $\mathcal{O}$ are the waves resulting from the Riemann problems solved at time $t=0$ at the discontinuities in $\bar{U}_{0}$.

Consider the Riemann problem for two neighboring states $U_{l}$ and $U_{r}$ of the piecewise constant data $\bar{U}_{0}$. Let the solution to the Riemann problem be

$$
\left(U_{l}, U_{r}\right)=\left[\left(U_{l}, U_{m}, U_{r}\right) /\left(\epsilon_{1}, \epsilon_{2}\right)\right] .
$$

Recall that $\epsilon_{1} \in[0, \pi)$ is the strength of the linear wave joining states $U_{l}$ and $U_{m}$ and is given by the angle between them. Since the states $U_{m}$ and $U_{r}$ are joined by a nonlinear wave, they must lie on the same radial line. Thus, $\epsilon_{1}$ is also the angle between states $U_{l}$ and $U_{r}$. We can derive the bound

$$
\left\|U_{r}-U_{l}\right\| \geq 2\left(\left\|U_{r}\right\|\left\|U_{l}\right\|\right)^{1 / 2} \sin \left(\epsilon_{1} / 2\right) \geq \frac{2 c_{1}}{\pi}\left|\epsilon_{1}\right| .
$$

Now let us find a similar bound for the wave $\epsilon_{2}$. Since the states $U_{m}$ and $U_{r}$ are joined by a nonlinear wave, the wave strength $\epsilon_{2}$ is given by $\epsilon_{2}=\Theta\left(U_{r}\right)-\Theta\left(U_{m}\right)$. Using the fact that states $U_{l}$ and $U_{m}$ are joined by 
a linear wave, we have $\epsilon_{2}=\Theta\left(U_{r}\right)-\Theta\left(U_{l}\right)$. Now using the smoothness of $\Theta$ and the fact that the data satisfies Equation (2), we obtain

$$
\left|\epsilon_{2}\right|=\left|\Theta\left(U_{r}\right)-\Theta\left(U_{l}\right)\right| \leq \max _{\|u\|<c_{2}}\|\nabla \Theta(u)\|\left\|U_{r}-U_{l}\right\| .
$$

Combining inequalities (12) and (13), we sum over all the discontinuities in piecewise constant $\bar{U}_{0}$ to obtain

$$
\left(\frac{\pi}{2 c_{1}}+\max _{\|u\|<c_{2}}\|\nabla \Theta(u)\|\right)\left\|\bar{U}_{0}\right\|_{T V} \geq \mathcal{L}(\mathcal{O}) .
$$

The lemma now follows from the fact that $\bar{U}_{0}$ is a piecewise constant approximation to the data $U_{0}$ and, hence, $\left\|U_{0}\right\|_{T V} \geq\left\|\bar{U}_{0}\right\|_{T V}$.

By Lemma 5.3, we have that $\mathcal{L}(\mathcal{O})$ is bounded whenever the total variation of the initial data is bounded. This in turn implies that $\mathcal{L}(J)$ is also bounded for any mesh curve $J$, because the functional $\mathcal{L}$ is monotone decreasing relative to the partial order on mesh curves. We employ this result to demonstrate that the constructed solutions $\left\{U_{h}\right\}$ are bounded as follows. Once we show that $\Theta\left(U_{h}\right)$ is bounded, we will show that the bounds on $U_{h}$ are attained on the level sets of the maximum and minimum values of $\Theta\left(U_{0}\right)$.

Lemma 5.4. Assume that $U_{0}$ is contained in an open half-space and satisfies (2). Then by the continuity of $\Theta$, there exist constants so that

$$
0=\Theta(0)<\Theta_{1} \leq \Theta\left(U_{0}\right) \leq \Theta_{2} .
$$

Define $\Omega \equiv\left\{r V: r>0\right.$ and $V=U_{0}(y)$ for some $\left.y \in \Re\right\}$. Then for all $(x, t), U_{h}(x, t) \in \Omega$, is contained in an open half-space, and satisfies

$$
0=\Theta(0)<\Theta_{1} \leq \Theta\left(U_{h}(x, t)\right) \leq \Theta_{2} .
$$

The proof follows from the analysis of the four possible Riemann solutions for the problem. Recall that for the possible Riemann problems, solutions remain in the half space and $\Theta$ is bounded by the initial data. That is to say that the solution, $U$, to the Riemann problem with left and right state $U_{L}$ and $U_{R}$, is such that $\Theta(U)$ is bounded above and below by $\Theta\left(U_{L}\right)$ and $\Theta\left(U_{R}\right)$. The fact that the Glimm approximate solutions are constructed from the solutions of Riemann problems at each time step gives the final result of the lemma.

From the above lemma we make the following important observation. Since $\Theta$ is both convex and coercive, the level sets of $\Theta$ at any given point are compact (see $[\mathbf{1 2}]$ ), and the following quantities can be defined:

$$
C_{1}=\min \left\{\|U\|: \Theta(U)=\Theta_{1}\right\},
$$

and

$$
C_{2}=\max \left\{\|U\|: \Theta(U)=\Theta_{2}\right\}
$$


Then for all $(x, t)$ it follows from the strict convexity of $\Theta$ that

$$
0<C_{1} \leq\left\|U_{h}(x, t)\right\| \leq C_{2},
$$

since $\Theta_{1} \leq \Theta\left(U_{h}(x, t)\right) \leq \Theta_{2}$.

We next show that the functional $\mathcal{L}$ is equivalent to the the total variation. Once we obtain this result we will be able to conclude that

$$
\left\|\left.U_{h}\right|_{J}\right\|_{T V} \leq \text { Const } \mathcal{L}(J) \leq \text { Const } \mathcal{L}(\mathcal{O}) \leq \text { Const }\left\|U_{0}\right\|_{T V},
$$

for any mesh curve $J$.

Lemma 5.5. If the initial data satisfies (15) and is contained in an open half-space, then $\mathcal{L}(\cdot)$ is equivalent to the total variation. Thus, there exist positive constants $B_{1}$ and $B_{2}$, independent of $U_{h}, J$, and the size of the mesh so that

$$
B_{1}\left\|\left.U_{h}\right|_{J}\right\|_{T V} \leq \mathcal{L}(J) \leq B_{2}\left\|\left.U_{h}\right|_{J}\right\|_{T V} .
$$

Proof. Let $J$ be any mesh curve. Note that the only places where $U_{h}$ (restricted to the mesh curve $J$ ) can change values is where some $\epsilon$-wave crosses the $J$-curve. Thus, the total variation of $U_{h}$, along a single $J$-curve, only increases at those places where a wave crosses $J$. In the following proof, we use result (16) which gives

$$
0<C_{1} \leq\left\|\left.U_{h}\right|_{J}\right\| \leq C_{2},
$$

whenever the initial data belongs to an open half-space. Suppose that a wave $\epsilon_{i}$ crosses $J$. Define $U_{l}$ to be the state to the immediate left of the wave and $U_{r}$ to be the state to the immediate right of the wave. Then there are only two possible cases:

(i) They are connected by a nonlinear wave $(i=2)$, or

(ii) they are connected by a linear wave $(i=1)$.

In each of the two cases, it will be shown that the contributions to $\mathcal{L}$ and $\|\cdot\|_{T V}$ satisfy (17).

(i) If the two states are connected by a nonlinear wave (or a 2-wave in this case), then it is a rarefaction or a shock. Since the two states are connected by a nonlinear wave, the states $U_{r}$ and $U_{l}$ must be on the same radial line and we can write $U_{r}=\left(\left\|U_{r}\right\| /\left\|U_{l}\right\|\right) U_{l}$.

Let

$$
D_{1}=\min \left\{\nabla \Theta(U) \cdot \frac{U}{\|U\|}:\|U\|=C_{1}\right\}
$$

and

$$
D_{2}=\max \left\{\nabla \Theta(U) \cdot \frac{U}{\|U\|}:\|U\|=C_{2}\right\}
$$


Using the assumption $\Theta$ is strictly convex with minimum at the origin, we obtain the desired bounds

$$
\frac{1}{D_{2}}\left|\epsilon_{2}\right| \leq\left\|U_{r}-U_{l}\right\| \leq \frac{1}{D_{1}}\left|\epsilon_{2}\right| \text {. }
$$

We now proceed to examine the case of a linear wave.

(ii) The two states $U_{l}$ and $U_{r}$ are connected by a linear wave. In this case $i=1$, and, by the definition of the wave strength, $\epsilon_{1}$ is the angle between $U_{l}$ and $U_{r}$. One can derive lower and upper bounds

$$
\frac{2 C_{1}}{\pi} \epsilon_{1} \leq\left\|U_{r}-U_{l}\right\| \leq\left|\left\|U_{r}\right\|-\left\|U_{l}\right\|\right|+2 C_{2} \epsilon_{1} .
$$

We observe that the argument is complete if $\left\|U_{l}\right\|=\left\|U_{r}\right\|$. If, however, $\left\|U_{l}\right\| \neq\left\|U_{r}\right\|$, an equivalence will need to be shown between the magnitude of the jump and the angle between the states.

Assume that $\left\|U_{l}\right\| \neq\left\|U_{r}\right\|$. From the mean value theorem, it follows that

$$
\left|\Theta\left(U_{l}\right)-\Theta\left(\left\|U_{l}\right\| \frac{U_{r}}{\left\|U_{r}\right\|}\right)\right| \leq 2 C_{2}\left\|\nabla \Theta_{\max }\right\| \epsilon_{1},
$$

where the quantity $\left\|\nabla \Theta_{\max }\right\|$ is defined to be

$$
\left\|\nabla \Theta_{\max }\right\|=\max _{\|U\| \leq C_{2}}\|\nabla \Theta(U)\| .
$$

Using the convexity of $\Theta$, it follows that

$$
D_{1}\left|\left\|U_{r}\right\|-\left\|U_{l}\right\|\right| \leq\left|\Theta\left(U_{r}\right)-\Theta\left(\left\|U_{l}\right\| \frac{U_{r}}{\left\|U_{r}\right\|}\right)\right| .
$$

The fact that the states $U_{l}$ and $U_{r}$ are connected by a contact discontinuity gives $\Theta\left(U_{l}\right)=\Theta\left(U_{r}\right)$, so that combining inequalities (19) and (20) yields

$$
\left|\left\|U_{r}\right\|-\left\|U_{l}\right\|\right| \leq 2 C_{2} \frac{\left\|\nabla \Theta_{\max }\right\|}{D_{1}} \epsilon_{1} .
$$

Hence, by inequality (18), we have for a discontinuity in the first characteristic field,

$$
\frac{2 C_{1}}{\pi} \epsilon_{1} \leq\left\|U_{r}-U_{l}\right\| \leq 2 C_{2}\left(1+\frac{\left\|\nabla \Theta_{\max }\right\|}{D_{1}}\right) \epsilon_{1} .
$$

Combining the results from cases (i) and (ii), the final result is obtained by summing over all jumps along mesh curve $J$ to obtain

$$
B_{1} \mathcal{L}(J) \leq\left\|\left.U_{h}\right|_{J}\right\|_{T V} \leq B_{2} \mathcal{L}(J) .
$$

Now using Lemmas 5.2 and 5.3, we obtain

$$
\left\|\left.U_{h}\right|_{J}\right\|_{T V} \leq B_{2} \mathcal{L}(J) \leq B_{2} \mathcal{L}(\mathcal{O}) \leq \frac{B_{2}}{B_{1}} \mathcal{K}\left\|U_{0}\right\|_{T V}
$$


This concludes the proof.

We have shown that Glimm's scheme produces a sequence $\left\{U_{h}\right\}$ which is uniformly bounded and has uniformly bounded total variation. Using Helly's theorem, this is sufficient to show that, for a fixed time $t,\left\{U_{h}(\cdot, t)\right\}$ has a convergent subsequence. In order to prove $\left\{U_{h}\right\}$ has a convergent subsequence, we require the following lemma. The lemma and its proof are from Smoller, Corollary 19.8 in [13], and follow from the fact that the total variation at any time is bounded by the total variation of the initial data; see Equation (23).

Lemma 5.6. Assume that $\Delta x / \Delta t$ satisfies the $C F L$ condition and that, in addition, $\Delta x / \Delta t<\lambda_{m}$. There exists a positive constant $C$, independent of $h$ such that

$$
\int_{-\infty}^{\infty}\left|u_{h}(x, t)-u_{h}\left(x, t^{\prime}\right)\right| d x \leq C\left|t-t^{\prime}\right| .
$$

\section{Existence of weak solutions.}

Using the previous results (16) and (23), which state that approximations generated by the Glimm scheme are bounded and that they are uniformly bounded in total variation, we show that the Glimm scheme has a convergent subsequence. The following classical theorem and proof showing that Glimm's scheme converges are due to Glimm [6].

Consider the net (a generalized sequence) $\left\{u_{h}\right\}$. Define the the indexing set so that $h=(\Delta x, \Delta t)$ and $(\Delta x) /(\Delta t)$ satisfies the CFL condition. The partial order " $\succ$ " on the index set is defined: If $i=\left(\Delta x_{i}, \Delta t_{i}\right)$ and $j=$ $\left(\Delta x_{j}, \Delta t_{j}\right)$, then $j \succ i$ when $\Delta x_{j} \leq \Delta x_{i}$ and $\Delta t_{j} \leq \Delta t_{i}$.

Theorem 6.1. If the net $\left\{u_{h}(x, t)\right\}$ satisfies:

(i) $\sup \left\|u_{h}(x, t)\right\| \leq C$,

(ii) $\stackrel{x}{\| u_{h}(\cdot, t)} \|_{T V} \leq M_{1}$, and

(iii) $\int_{\Re}\left|u_{h}(x, t)-u_{h}(x, s)\right| d x \leq M_{2}|t-s|$,

then $u_{h}$ has a subnet which converges in $L_{\text {loc }}^{1}\left(\Re \times \Re^{+}\right)$.

In the proof, hypotheses (i) and (ii) imply Helly's theorem holds for any rational time $t_{i}$. Using a diagonalization process, we can show the existence of a subnet which converges for all rational time. Using the fact that the rationals are dense in the reals, the last hypothesis shows that the subnet converges for all real time.

Hence, results (16) and (23), along with Lemma 5.5, give the convergence of Glimm's scheme. Now, we show that the limit $u$ is a weak solution. For 
this, we must show that

$$
\iint\left(u \phi_{t}+f(u) \phi_{x}\right) d x d t+\int u_{0} \phi(x, 0) d x=0,
$$

for any test function $\phi \in C_{0}^{\infty}$. Assume that the support of $\phi$ is contained in $I \times[0, T]$, then by the Lebesgue Dominated Convergence Theorem, we have

$$
\iint u_{h} \phi_{t} \rightarrow \iint u \phi_{t}
$$

Similarly, this implies the limit $u$ is a weak solution if

$$
\iint\left(u_{h} \phi_{t}+f\left(u_{h}\right) \phi_{x}\right) d x d t+\int u_{0} \phi(x, 0) d x \rightarrow 0 .
$$

We now make the dependence on the random sequence $\left\{\theta_{i}\right\}$ explicit. Define

$$
J(\theta, h, \phi) \equiv \iint\left(u_{h}^{\theta} \phi_{t}+f\left(u_{h}^{\theta}\right) \phi_{x}\right) d x d t+\int u_{0} \phi(x, 0) d x .
$$

Once convergence of $J$ to 0 is established for the sequence $\left\{u_{h}^{\theta}\right\}$ obtained by Glimm's scheme, then the function $u(x, t)$ for which $u_{h} \rightarrow u$ will be a weak solution of the original conservation law (1).

Let $\mathcal{N}$ denote the set of natural numbers. Consider the product space $\Omega=(-1,1)^{\mathcal{N}}$, where $\left\{\theta_{i}\right\} \in(-1,1)^{\mathcal{N}}$. We endow the space $\Omega$ of sequences $A=\left\{a_{i}\right\} \in(-1,1)^{\mathcal{N}}$, with probability measure $d \nu(A)$. This is accomplished by normalizing, so $d \nu\left(a_{i}\right)$ equals half of the Lebesgue measure. Then the key result stated and proved by Glimm $[6]$ is:

Lemma 6.2. $\int_{\Omega}|J(A, h, \phi)|^{2} d \nu(A) \rightarrow 0$ as the mesh size goes to zero.

The proof is now complete, and we have shown that the approximations generated by Glimm's scheme converge to a weak solution for (1).

\section{Conclusions.}

In summary, we have shown that there exists a weak solution to the multiply characteristic conservation law system (1). Since the Riemann solutions exist for all data in an open half-space, we do not restrict the total variation of the initial data to be small as Glimm required in his original proof. Following the Glimm construction of approximate solutions, we showed that the approximate solutions satisfy the hypotheses of Helly's Theorem. As in Glimm's seminal paper, this was accomplished by showing the constructed solutions were bounded in an equivalent functional. It is in the choice of the functional that the method used in this paper diverges from the literature. This was a necessary modification, since the standard choice of an equivalent functional (as in Glimm [6]) is not appropriate for conservation laws possessing multiple characteristics. With our chosen functional, we show that 
wave interactions decouple, which gives the result that wave interactions are monotone decreasing on the partial order of mesh curves. After establishing that the total variation norm is equivalent to the functional, the remainder of the existence proof, that the limit of the subsequence is a weak solution, is identical to Glimm's proof of existence for strictly hyperbolic conservation laws.

\section{References}

[1] G.-Q. Chen and H. Frid, Existence and asymptotic behavior of measure-valued solutions for degenerate conservation laws, J. Differential Equations, 127 (1996), 197-224, MR 97d:35131, Zbl 0854.35066.

[2] J.L. Doob, Measure Theory, Springer-Verlag, New York, 1994, MR 95c:28001, Zbl 0791.28001.

[3] H. Freistühler, Linear degeneracy and shock waves, Mathematische Zeitschrft, 207 (1991), 583-596, MR 92e:35108, Zbl 0756.35048.

[4] - On the Cauchy problem for a class of hyperbolic systems of conservation laws, J. Differential Equations, 112 (1994), 170-178, MR 95i:35179, Zbl 0806.35113.

[5] H. Freistühler and E.B. Pitman, A numerical study of a rotationally degenerate hyperbolic system. I. The Riemann problem, J. Comput. Phys., 100 (1992), 306-321, MR 93a:65132, Zbl 0757.65096.

[6] J. Glimm, Solutions in the large for nonlinear hyperbolic systems of equations, Comm. Pure Appl. Math., 18 (1965), 95-105, MR 33 \#2976, Zbl 0141.28902.

[7] V. Guillemin and A. Pollack, Differential Topology, Prentice-Hall, Inc., New Jersey, 1974, MR 50 \#1276, Zbl 0361.57001.

[8] B.L. Keyfitz and H.C. Kranzer, A system of non-strictly hyperbolic conservation laws arising in elasticity theory, Arch. Rational Mech. Anal., 72 (1980), 219-241, MR 80k:35050, Zbl 0434.73019.

[9] , The Riemann problem for a class of hyperbolic conservation laws exhibiting a parabolic degeneracy, J. Differential Equations, 47 (1983), 35-65, MR 84a:35162, Zbl 0521.35035.

[10] P.D. Lax, Hyperbolic systems of conservation laws, II, Comm. Pure Appl. Math., X (1957), 537-566, MR 20 \#176, Zbl 0081.08803.

[11] R.J. LeVeque, Numerical Methods for Conservation Laws, Birkhäuser, Boston, 1992, MR 92m:65106, Zbl 0847.65053.

[12] R.T. Rockafellar, Convex Analysis, Princeton University Press, Princeton, New Jersey, USA, 1970, MR 43 \#445, Zbl 0193.18401.

[13] J.A. Smoller, Shock Waves and Reaction-Diffusion Equations, Springer-Verlag, New York, 1983, MR 84d:35002, Zbl 0508.35002.

Received February 2, 2000 and revised November 15, 2001.

Division of Mathematical and Computational Sciences

National Institute of Standards and Technology

Gaithersburg, MD 20899-8910 
E-mail address: anthony.kearsley@nist.gov

Department of Applied Mathematics

Illinois Institute of TEChNOLOGY

Chicago, IL 60616

E-mail address: reiff@iit.edu 